\title{
Short fifth metacarpals-insulin resistance syndrome
}

INSERM

\section{Source}

INSERM. (1999). Orphanet: an online rare disease and orphan drug data base. Short fifth metacarpals-insulin resistance syndrome. ORPHA:66518

Short fifth metacarpals-insulin resistance syndrome is characterised by bilateral shortening of the fifth fingers and fifth metacarpals. It has been described in several members of one family. Some members of the family also had spherocytosis and insulin resistance. Transmission is autosomal dominant. 